\title{
CONOCIMIENTO PROFESIONAL DOCENTE DEL PSICÓLOGO. UN ESTUDIO DE CASO
} PROFESSIONAL KNOWLEDGE OF THE PSYCHOLOGIST. A CASE STUDY

\author{
Adriana M. Rodríguez ${ }^{1}$ - Margarita C. Ortiz ${ }^{2}$
}

Fecha de recepción: 11-05-2018

Fecha de aceptación y versión final: 19-07-2018

Resumen: El Conocimiento Profesional Docente (CPD) es un proceso complejo que requiere procesos reflexivos para afrontar los problemas de enseñanza y de aprendizaje que enfrentan en el ejercicio de la docencia los Psicólogos y Licenciados en Psicología de la Licenciatura en Psicología de una universidad del nordeste argentino.

Numerosos estudios concluyen que la enseñanza de la Psicología en este ámbito, generalmente asume la modalidad tradicional basada en el modelo didáctico de la trasmisión. Con el objetivo de promover procesos de cambio e innovación en las prácticas pedagógicas e institucionales, se indaga el CPD focalizando una de las tareas del docente universitario: la enseñanza.

Para ello, entrevistamos a una muestra de profesores principiantes y experimentados -de una de las sedes de la carrera- calificados como casos de "buenas prácticas de enseñanza", por sus alumnos, en una encuesta. Los entrevistados denotan rasgos personales y profesionales como "saber la materia y saber enseñarla" que sintetizamos en los siguientes indicadores: logros de los estudiantes, su satisfacción con la enseñanza brindada y pasión por la enseñanza.

Palabras clave: Docentes universitarios- buenas prácticas- enseñanza

Abstract: The Professional Teaching Knowledge (CPD) is a complex process that requires reflexive processes to face the teaching and learning problems faced by the Psychologists and Graduates in Psychology of the Bachelor of Psychology of a university in northeastern Argentina.

Numerous studies conclude that the teaching of psychology in this area generally assumes the traditional modality based on the didactic model of transmission. With the objective of promoting processes of change and innovation in pedagogical and institutional practices, the CPD is investigated, focusing on one of the tasks of the university teacher: teaching.

To do this, we interview a sample of beginner and experienced teachers - from one of the venues of the career - classified as cases of "good teaching practices", by their students, in a survey. The interviewees denote personal and professional traits such as "knowing the subject and knowing how to teach it" that we synthesize in the following indicators: student achievements, their satisfaction with the teaching provided and passion for teaching.

Key words: University teachers- good practices- teaching

\footnotetext{
${ }^{1}$ Lic. en Psicología. Especialista en Salud Social y Comunitaria y Metodología de la investigación. Coordinadora de la Licenciatura en Psicología. Prof. Asociada de Metodología de la Investigación y de Trabajo Integrador Final de la Facultad de Psicología, Educación y Relaciones Humanas. Universidad de la Cuenca del Plata. Prof. Adjunta en Psicología del niño. Profesorado y Licenciatura en Educación Inicial. Facultad Humanidades. UNNE.

2 Prof. en Biología. Mgter en Epistemología y Metodología de la Investigación Científica. Especialista en Investigación Educativa y Docencia Universitaria. Doctoranda en Cs. Cognitivas. Prof. Adjunta de Neurociencias I- Licenciatura en Psicología de la Universidad de la Cuenca del Plata y Prof. Adjunta Reg. de Teoría y Métodos de la Investigación. Facultad de Humanidades. UNNE.
} 


\section{Introducción}

En el imaginario docente universitario hasta hace unas décadas se entendía que para enseñar era suficiente conocer la disciplina objeto de enseñanza. En este contexto, la experticia disciplinar habilita enseñarla. Supuesto que da cuenta de la docencia concebida como actividad sencilla que cualquier profesional puede desempeñar. Ya en la década del '90, Sancho Gil y Hernández Hernández, en su obra "Para enseñar no basta con saber la asignatura" alertan acerca de los modos de abordar la relación entre lo que el profesor pretende enseñar y lo que el alumno puede aprender.

Dada la supremacía del conocimiento disciplinar por sobre los conocimientos pedagógicos y didácticos aspectos en la docencia universitaria, la agenda de la educación superior contempla programas específicos de formación y capacitación docente, tales como Profesorado Universitario, Especializaciones en Docencia Superior/Universitaria, entre otros.

La enseñanza de la Psicología en la Licenciatura en Psicología es uno de los focos de preocupación de la Coordinación de la carrera, debido a algunos señalamientos estudiantiles en la evaluación de sus profesores, entre los que cabe destacar:

Tensión teoría -práctica reflejada en la prevalencia de la dimensión conceptual disciplinar por sobre su transferencia en la resolución de problemas concretos de la práctica profesional, con escaso vínculo con las competencias del egresado para una efectiva inserción laboral.

Tensión entre el conocimiento disciplinar y el conocimiento pedagógico y didáctico expresada en el limitado uso de estrategias de enseñanza y de evaluación y de materiales curriculares.

Escaso impacto de las acciones de formación permanente en los Psicólogos profesionales que se desempeñan en las unidades académicas de las localidades de Corrientes, Misiones, Formosa, Chaco y Santa Fe, debido al asiduo cambio o renovación de docentes de las cátedras.

No obstante, los alumnos coinciden en enfatizar la existencia de "buenas prácticas de enseñanza" de algunos/as Psicólogos/as.

En este sentido, el trabajo de Carlos Guzmán (2009) ¿Cómo enseñan psicología los profesores efectivos? Un estudio exploratorio (UNAM, México), constituyó un antecedente del proyecto de investigación sobre el Conocimiento Profesional Docente de los Psicólogos y Licenciados en Psicología, con el propósito de buscar alternativas de mejorar de las prácticas docentes en esta carrera.

Trabajamos con docentes con estas titulaciones, valorados por sus alumnos como ejemplos de "buenas prácticas de enseñanza", a fin de caracterizar éstas, su pensamiento didáctico y las prácticas docentes que dicen realizar. 


\section{Encuadre conceptual}

Las universidades están sujetas a transformaciones (Zabalza, 2006) por lo que los profesores continuamente enfrentan desafíos, que actualmente implican una enseñanza centrada en el aprendizaje para desarrollar competencias profesionales y transversales y la autonomía de los estudiantes en su proceso de aprendizaje.

Los docentes universitarios están formados en el conocimiento especializado de un determinado campo disciplinar. Comienzan a construir un conocimiento declarativo en torno a la disciplina en estudio (Medina Moya, 2006; Marcelo, 2009) en el trayecto formativo en la Universidad. Es un conocimiento explícito y teórico que no requiere ser validado experiencialmente (Torres, 1998) y refiere al "saber qué enseñar". Constituye el sustrato para la construcción del saber profesional docente.

El conocimiento profesional docente (CPD), en tanto constructo se nutre de variadas fuentes: experiencias de vida, de formación académica y de socialización profesional. Se reelabora, resignifica y transforma en los diversos espacios de actuación de los/as docentes.

EI CPD es proceso y producto a la vez e involucra la construcción y apropiación de saberes que permiten actuar conforme requerimientos de la práctica. Es un conocimiento ligado a la acción, a la experiencia, e incluye en su configuración otros tipos de conocimiento construidos durante la formación inicial y permanente y en la socialización profesional.

La formación profesional refiere al conjunto de procesos de preparación de un sujeto, para un posterior desempeño en el ámbito laboral/profesional, que se lleva cabo instituciones de educación superior y apunta la formación de competencias transversales y específicas en un campo disciplinar/profesional. (Fernández Pérez, 2001)

Los docentes universitarios tienen que dominar el contenido de la materia de la disciplina que enseñan y poseer un conocimiento acerca de cómo enseñarlo, esto es, el CDC-conocimiento didáctico del contenido-(Shulman, 1987). Según el autor, este tipo de conocimiento permite diferenciar a especialistas de docentes y a los últimos, explicar los tópicos de sus disciplinas de modo que los estudiantes puedan comprenderlos. Es un conocimiento que se aprende y requiere, además, la capacidad para reflexionar en torno a los propios campos de saber disciplinar y sus prácticas pedagógicas, en un proceso de meta-aprendizaje. (Schön, 1982)

Entendemos que los procesos de metacognición favorecen la toma de contacto con ideas, imágenes, representaciones que guían el conocimiento profesional, la manera de percibir, interpretar y resolver los problemas prácticos profesionales, así como la toma de conciencia de contradicciones que surgen a partir de la confrontación entre éstos. Dichos procesos implican revisión, reelaboración y re-significación de los conocimientos construidos y de los procesos cognitivos involucrados en el desarrollo conceptual y el aprendizaje de la práctica profesional.

En tal sentido, constituye un tipo de reflexión específica, esto es, sobre la propia cognición, (Sanjurjo, 2004), posibilitando a los/as docentes explicitar los fundamentos de sus proyectos y acciones y su reformulación. La reconstrucción del conocimiento, a través de estos procesos, promueve prácticas significativas, la articulación de saberes y la toma de conciencia del papel mediador de discursos dominantes sobre la acción y el pensamiento (Feldman, 1999: 101) 
Ahora bien, la mayoría de Psicólogos y Licenciados en Psicología que ejercen como profesores universitarios recibieron escasa formación pedagógica o carecen de ella y como principiantes en la docencia - primeros cinco años- articulan recursivamente el conocimiento declarativo que poseen con las prácticas docentes. (Schön, 1992; Medina Moya, 2006; Marcelo, 2009)

\section{Pensamiento didáctico y Buenas prácticas de enseñanza}

Concebimos al pensamiento didáctico del profesor como el conjunto de concepciones acerca de temas propios de su actividad: la finalidad de sus acciones, los modos de concebir la enseñanza, el aprendizaje, la evaluación y a los propios alumnos; resultante de procesos de formación inicial y continua y de la experiencia docente. Incluye pensamientos, creencias, visiones, actitudes y concepciones de los/as profesores/as en torno a su labor y la descripción que hacen de su práctica docente. Dado que son los supuestos subyacentes que guían y justifican las acciones realizadas y determinan-en cierta medida- lo que hacen, su estudio permite entender y comprender la docencia.

El conocimiento del pensamiento de los buenos docentes en las aulas universitarias puede ser útil para la mejora de la enseñanza en este contexto (Korthagen, 2001; Kane, et al., 2002), pues los cambios requieren del examen y transformación de las ideas sobre ésta.

La clase- estructurante de la práctica docente- es el “(...) espacio pedagógico, de poder, de comunicación, de relaciones, de orden y desorden, de consenso y conflicto, de encuentro social a la vez que epistemológico". (Sanjurjo, 2012: 79)

En el escenario áulico, la noción de enseñanza alude a significados diversos, tal como señala Davini (2009):

1. Es una acción intencional de transmisión cultural y en este sentido, refiere a las acciones de:

$\checkmark$ Transmitir conocimientos o saberes.

$\checkmark$ Favorecer el desarrollo de una capacidad.

$\checkmark$ Corregir y apuntalar una habilidad.

$\checkmark$ Guiar una práctica

2. En tanto mediación social y pedagógica implica:

$>$ Vincular los contenidos generales a enseñar con las necesidades, idiosincrasia y la cultura del grupo.

$>$ Proponer actividades, discusiones, profundizaciones, ejercicios que favorezcan el tratamiento de los contenidos, teniendo en cuenta necesidades, intereses y cultura de los/as estudiantes.

> Favorecer el intercambio a partir de expectativas y concepciones.

$>$ Ampliar conocimientos y perspectivas particulares, brindando información, otros puntos de vistas y experiencias.

Vincular la enseñanza al contexto particular y a las situaciones específicas.

3. Es un sistema de relaciones e interacciones reguladas entre sujetos con roles diferenciados que supone la existencia de:

- Alguien que conoce lo que enseña y confía en la posibilidad de aprender de los/as otros/as.

- Alguien que acepta participar de la enseñanza. 
- Una asignatura, materia o contenido a enseñar, considerado válido y valioso de ser aprendido.

- Un ambiente que facilite el desarrollo de la enseñanza y del aprendizaje.

4. Configura una secuencia metódica de acciones:

- No ocurre de modo espontáneo.

- Es una actividad sistemática.

- Implica un proceso interactivo entre los/as participantes, según valores y resultados que se busca alcanzar.

Recapitulando, la enseñanza es una acción intencional, voluntaria y conscientemente dirigida para que otro/s aprenda/n lo que no puede/n aprender solo/s, de modo espontáneo o por sus propios medios. Tal como expresa Fenstermarcher (1989), la tarea del profesor no consiste en transmitir un contenido, sino permitir que los estudiantes tomen posesión de éste donde quiera que se halle.

Si bien entendemos que enseñar es ayudar a aprender, destacamos que los procesos de enseñanza difieren en calidad o idoneidad práctica. En este sentido, en la nueva agenda didáctica se indaga acerca de las buenas prácticas y buena enseñanza.

Aunque de una semántica borrosa, el concepto de buenas prácticas en general está en el núcleo de la investigación educativa desde hace mucho tiempo. De acuerdo con Van Haecht (1998), la búsqueda de referentes de buenas prácticas e instituciones modélicas figuraba en escritos de inicios del S.XIX. La idea de buenas prácticas aparece vinculada a las de innovación, calidad, evaluación, entre otras y forma parte del repertorio de conceptos de políticas sociales y educativas.

En la identificación de buenas prácticas se busca modalidades diversas de responder, con eficacia y satisfacción de los participantes, a las diferentes demandas (en este caso educativas) del contexto (Benavente, 2007). En ese sentido, el concepto de buenas prácticas es aplicable a cualquier ámbito de la actividad humana, desde las políticas hasta las acciones específicas de sujetos individuales.

Dos características fundamentales son de destacar cuando se afronta una investigación bajo el paraguas semántico de las buenas prácticas:

- La bidimensionalidad del concepto de práctica. Las teorías de la acción del grupo Harvard (1950) señalan que ésta es una realidad objetiva y subjetiva a la vez, es algo que se hace y algo que tiene un sentido, un acto personal y una realidad cultural.

- El concepto de buenas prácticas se enriquece en aquellas formulaciones en las que la práctica se transforma en praxis. Este concepto incluye componentes éticos y orienta la práctica a la mejora de las situaciones en las que se analiza. Praxis que, en términos de Freire (1970), es reflexión y acción sobre el mundo para transformarlo. Una buena práctica mejora el statu quo de las cosas y de las personas. La praxis tiene por objetivo transformar la realidad y mejorarla.

La literatura anglosajona refiere al concepto de mejores prácticas que tiene, a su vez, una cualidad relativizadora, es lo mejor que se puede hacer en un determinado marco de condiciones, lo inmejorable, algo contextualizado.

Los principales problemas que plantea el concepto de buena práctica como punto de partida para la investigación pertenece a dos órdenes de decisiones: 1) los ámbitos sobre los que se desea analizarla y 2) los criterios que permiten identificar una acción como buena práctica con suficiente rigor y fiabilidad. 
Por su parte, la buena enseñanza Porta y Sarasa (2008) refiere a las buenas acciones docentes, identificadas por la intuición, la sabiduría práctica y la espontaneidad. La buena enseñanza no se define por su éxito en términos de buenos resultados de aprendizaje (los suponen), sino por su fuerza moral y por su fuerza epistemológica (Fenstermarcher,1989).Se preocupa y promueve aprendizajes profundos involucrando a los estudiantes en actividades desafiantes, que despiertan curiosidad y se concreta en docentes que tienen: "... la habilidad de ocupar a sus estudiantes en algo que les resultará fascinante porque es tan inesperado y porque alguien los considera con seriedad" (Bain, 2012:72).

\section{Metodología}

Nos posicionamos en el enfoque cualitativo y el paradigma interpretativo con el objetivo de aproximarnos a la comprensión del conocimiento y las prácticas de Psicólogos/as que ejercen la profesión docente.

La estrategia metodológica es el estudio de casos (Stake, 1994) con múltiples instrumentos de recolección de información empírica: entrevistas en profundidad; observaciones no participantes de clases y materiales curriculares.

En esta oportunidad trabajamos con 14 docentes (Psicólogos y Licenciados en Psicología) principiantes y experimentados a cargo de asignaturas específicas o de orientación al campo profesional de la carrera Licenciatura en Psicología de la mencionada unidad académica, a los/as que entrevistamos.

Dichos profesores/as fueron seleccionados como ejemplos de buenas prácticas de enseñanza por una muestra de estudiantes $(n=55)$, a quienes aplicamos un cuestionario.

En el siguiente cuadro discriminamos los 14 profesores distinguidos sobre la base del nivel o curso en los que se desempeñan y porcentual de selección alcanzado.

Cuadro N¹: Casos de Buenas prácticas de enseñanza

\begin{tabular}{|l|l|l|l|l|l|}
\hline \multirow{2}{*}{ Porcentaje } & \multicolumn{5}{|l|}{ Cantidad de docentes seleccionados por curso } \\
\cline { 2 - 6 } & $1^{\circ}$ año & $2^{\circ}$ año & $3^{\circ}$ año & $4^{\circ}$ año & $5^{\circ}$ año \\
\hline 50 a $70 \%$ & 1 & 2 & 1 & 1 & 1 \\
\hline 29 al $49 \%$ & 1 & 1 & 2 & 2 & 2 \\
\hline
\end{tabular}

Fuente: Cuestionario de estudiantes.

La mayoría de los/as profesores/as (Psicólogos y Licenciados en Psicología) acredita una antigüedad en la carrera entre 2 y 10 años y dos de ellos, más de 10 años. Cinco son principiantes (1 a 5 años de ejercicio docente) El $40 \%$ realizó posgrados en su campo profesional (Especializaciones y Maestrías) y los menos (20\%), Especialización en Docencia Universitaria o en Metodología de la Investigación. 
Asimismo, también focalizamos la mirada en una de las cátedras de $2^{\circ}$, para lo cual analizamos actas acuerdos de 2017 y 2018 realizadas entre el profesor de la sede y los de equipos docentes de las restantes, de la asignatura elegida, su programación, muestras de trabajos prácticos, evaluaciones parciales, portfolios de los/as estudiantes e informe final individual. Estos instrumentos dan cuenta del pensamiento del profesor, de los supuestos subyacentes en las decisiones y de las reflexiones metacognitivas en torno a los procesos de enseñanza y de aprendizaje, logros alcanzados en tanto aprendizajes construidos.

En suma, tratamos de definir dispositivos que permitiera al colectivo profesional docente "explicitar el conocimiento y convertirlo en experiencias susceptibles de análisis personal” (Diker y Terigi,2003:109).

\section{Presentación y discusión de resultados}

I. El contexto de estudio

La Licenciatura en Psicología es una carrera que se dicta en 4 sedes de la Universidad de referencia. El estudio exploratorio-descriptivo se realizó en una de ellas.

Las unidades académicas de cada sede cuentan con salas climatizadas, equipamiento informático y otros recursos y materiales curriculares disponibles en la Biblioteca Especializada y Sala de Informática.

El cuerpo docente de la carrera está constituido por profesionales con distinta titulación: Psicólogos, Licenciados en Psicología, Médicos Neurólogos, Médicos Psiquíatras, entre otros.

Los docentes y coordinadores de todas las sedes se mantienen en permanente contacto y comunicación. Los equipos docentes unipersonales lo hacen para consensuar la planificación de la materia a cargo conforme un formulario base y un acta acuerdo institucional, vía skype.

El plan de estudio consta de 35 materias distribuidas en dos ciclos: 1) Ciclo de Orientación que abarca $1^{\circ}$ y $2^{\circ}$ años y 2) Ciclo de Profesionalización que incluye de $3^{\circ}$ a $5^{\circ}$ año.

De los 35 docentes, mostramos aquellos que ostentan titulación de Psicólogos o Licenciados en Psicología, en 25 materias de ambos ciclos:

Cuadro N²: Psicólogos o Licenciados en Psicología a cargo de Cátedras

\begin{tabular}{|l|c|c|c|c|c|}
\hline Ciclos & \multicolumn{2}{|c|}{ Orientación } & \multicolumn{4}{|c|}{ Profesionalización } \\
\hline Cursos & $1^{\circ}$ & $2^{\circ}$ & $3^{\circ}$ & $4^{\circ}$ & $5^{\circ}$ \\
\hline Cantidad & 2 & 6 & 6 & 8 & 3 \\
\hline
\end{tabular}

Fuente: Plan de estudio

La mayoría de estos profesionales ejerce la profesión de modo independiente (consultorios privados) y/o dependientes en consultorios públicos en establecimientos del Estado (Centros de Salud Mental, Hospitales, Empresas, Asilos, entre otros). 
Cada profesor/a tiene a cargo un grupo de aprendizaje que oscila entre 30 y 15 estudiantes, procedentes de capitales y localidades del interior de dos provincias vecinas.

II. Lo que dicen los estudiantes

Presentamos los resultados de los cuestionarios aplicados a la muestra de 55 estudiantes de la sede seleccionada.

\section{Profesores seleccionados como casos de buenas prácticas de enseñanza}

Los estudiantes describen como buenas prácticas de enseñanza a situaciones en las que aprendieron "cosas" que no olvidaron, realizaron actividades motivadoras e interesantes en las que asumieron futuros roles profesionales, trabajaron con casos concretos de la práctica profesional, tuvieron un papel activo y fueron protagonistas de sus aprendizajes.

Rasgos de los docentes caracterizados por sus buenas prácticas de enseñanza

Entre los rasgos personales de los docentes seleccionaron palabras de las cuales, las más recurrentes son: vocación; responsabilidad; compromiso; buen trato; claridad; didáctica.

Para Sánchez Blanco (2003), la vocación es el motor que dinamiza la enseñanza, es una condición sine qua non para el bien ejercicio de ésta:

"Aman lo que hacen, tienen vocación docente y son organizados en sus clases"(Enc. 38-4año).

"Se nota la vocación que tienen, hacen que te intereses por los temas (Enc.33$\left.3^{\circ} a \tilde{n} 0\right)$.

Para Litwin (2008), la vocación docente es un elemento en ocasiones olvidado en estudios didácticos al momento de entender la práctica de la enseñanza, posiblemente por su implicancia en la tarea docente. No obstante, algunas indagaciones señalan que el docente con vocación de enseñanza reconstruye narrativas significativas con sus alumnos y emplea la escucha sensible, comprensión y compasión que son sus inquietudes en el acto de enseñar (Litwin, 2008 citada en Alvarez y Sarasa, 2007).

Por otra parte, el trato hace al vínculo pedagógico docente-estudiantes sustentado en el respeto mutuo, la confianza del profesor para compartir hitos de sus trayectorias formativas y animarlos a reflexionar (Bain, 2007):

"Tienen buen trato con nosotros y no se molestan cuando preguntamos, te ayudan a aprender" (Enc.11-1 ${ }^{\circ}$ año).

"Tienen mucha responsabilidad y compromiso con lo que hacen" (Enc.24-2。 año).

"Nos tienen confianza y nos cuentan sus errores y temores de cuando eran estudiantes para hacernos reflexionar" (Enc. 53-5 año).

La clave para comprender a los mejores docentes está en sus actitudes, en su fe en la capacidad de logro de sus estudiantes, en su predisposición a tomarlos en serio y dejar que asuman el control sobre su propia formación, y en su compromiso en 
conseguir que los criterios y prácticas surjan de objetivos de aprendizaje cardinales y del respeto y acuerdo entre estudiantes y profesores (Bain, 2007:92).

Los rasgos subrayados como características de buena enseñanza: claridad, estimulación del interés e involucramiento de los estudiantes para el aprendizaje en clase, son consistentes con los hallazgos de Hativa (2000) y Carlos Guzmán (2009).

La claridad contribuye al desarrollo de la capacidad de comprensión de los contenidos enseñados, entendiendo ésta como su utilización en nuevas situaciones.

"Se destacan por la claridad al explicar y la didáctica de sus clases (Enc.49-3 año).

"Tienen didáctica para dar la clase, saben llegar para que entiendas mejor los temas" (Enc.20 -2ªño).

La claridad- en tanto componente de buena enseñanza-, favorece los aprendizajes de los estudiantes.

Los docentes distinguidos muestran un alto compromiso con la comunidad académica y conciben la evaluación como una herramienta para ayudarlos y motivarlos a aprender (Bain, 2004).

"Son exigentes pero tienen flexibilidad en los exámenes y buscan diferentes maneras de comprobar si aprendimos"(Enc.31-3año).

"Se preocupan para que sus alumnos aprendamos la materia y si comprendimos lo que enseñan” (Enc.44-4ªño).

Estos rasgos destacados por los alumnos dan cuenta de docentes con concepciones de enseñanza centradas en los alumnos y sus aprendizajes.

Entre los rasgos profesionales de los profesores reconocidos por sus estudiantes como buenos docentes, destacan la autoridad pedagógica, esto es, saben mucho de su materia y transmiten pasión por el conocimiento que enseñan:

"Cuando enseñan ponen mucha pasión y eso se siente, relacionan lo que damos con lo que haremos al recibirnos" (Enc. $47-4^{\circ}$ año).

"Transmiten todo lo que saben con claridad, tienen mucha didáctica y te ayudan a aprender lo que enseñan, comprender los temas” (Enc.27-3ªño).

"Preparan muy bien sus clases y las evaluaciones...bueno...esas son las materias que más nos sirven cuando estamos llegando al final" (Enc.51- 5 año).

"Trabajan con muchos recursos con actividades concretas, te hacen relacionar lo que damos en el teórico con la práctica y así comprendemos mejor lo que enseñan y lo que necesitamos para ejercer la profesión (Enc. $48-5^{\circ}$ año).

Los estudiantes señalan la variedad de técnicas que ponen en juego los docentes en sus clases, de recursos y materiales que median la enseñanza y las actividades que posibilitan la transferencia de los aprendizajes a situaciones de la realidad.

La organización es otra de las cualidades o rasgos identificados relacionada con los modos de presentar los contenidos, la ejemplificación, la claridad al explicar, la 
capacidad para responder dudas e interrogantes, enfatizar lo importante, sintetizar lo enseñado y evaluar el nivel de comprensión alcanzado (Carlos Guzmán,2009).

El saber erudito sobre la disciplina es visibilizado por los estudiantes como autoridad pedagógica, saber la materia e "investigar los problemas del campo disciplinar /profesional, pasión que transmiten en sus clases" (Enc. 54, $5^{\circ}$ año).

Estos profesores además de saber, saben enseñar, lo que se observa en el modo de presentar los temas de sus materias, tienen predisposición para explicar "tantas veces como sea necesario", paciencia para responder las preguntas, evalúan con rigor y flexibilidad. De este modo, lo disciplinar y lo pedagógico se complementan, se articulan la forma y el contenido.

Denotan dominio del campo disciplinar y habilidad para hacer comprensibles temas complejos, conocimientos acerca del aprendizaje y gusto por la enseñanza. Se preocupan por el aprendizaje de sus estudiantes generando un clima para que éste se logre, con los apoyos y mediaciones necesarios y confiando en sus capacidades, tal como expresa Bain (2004).

Aunque los rasgos personales y profesionales se solapan en el discurso de los estudiantes, los rasgos seleccionados difieren según el ciclo de la carrera. Los del ciclo básico priorizan las cualidades personales por sobre las características profesionales, mientras que los del ciclo superior, mostraron una tendencia hacia las últimas.

En suma, los rasgos de Psicólogos y Licenciados en Psicología con buenas prácticas de enseñanza, pueden sintetizarse en los siguientes indicadores: logros de los estudiantes y su satisfacción con la enseñanza brindada (Hativa, 2000).

\section{Lo que dicen los profesores}

En este apartado presentamos las voces de los/as profesores/as reconocidos por sus alumnos como figuras clave en su trayecto de formación como Licenciados en Psicología.

Exponemos los resultados de las entrevistas conforme las dimensiones de análisis.

\section{III.1.Características de docentes con "buenas prácticas de enseñanza"}

Los profesores seleccionados aluden a figuras y espacios que constituyen referencias en su formación como docentes universitarios. Recuerdan que dichos referentes sabían mucho de sus materias y mostraban pasión por el conocimiento que enseñaban. Los/as docentes reconocen a dichos referentes como modelos que admiraban y a la vez, temían (Freud,1982). Las figuras que incluyeron, dejaron marcas y de éstas tomaron algunos de los rasgos que los estudiantes detectaron en sus prácticas. No obstante, reconocen que no existe una única forma de actuar sino muchas. (Jackson, 2002). Además de estas figuras, relatan que algunas de sus vivencias personales, escolares y profesionales contribuyeron a la configuración de su sabiduría práctica. Se trata de docentes experimentados comprometidos con la tarea de enseñar, apasionados por lo que hacen e inquietos por encontrar diferentes formas de hacerlo. 
En el siguiente esquema se exponen las sub-dimensiones de las "buenas prácticas de enseñanza" de los/as docentes noveles y experimentados destacados:

Características de docentes con "buenas prácticas de
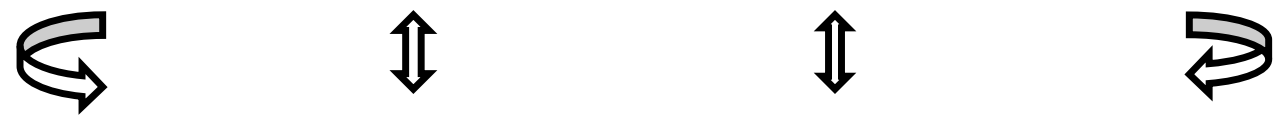

\section{Motivación}

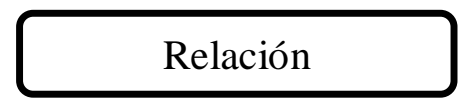

Compromiso

Responsabilidad

Los/as profesores conciben la motivación como los modos de dar respuesta a las expectativas de sus estudiantes acerca de cómo realizarán las diferentes actividades vinculadas con la futura práctica profesional y las razones que los y las movilizan a cumplimentarlas. Esta concepción es consistente con modelos de comportamiento expectación-valor. (Eccles y Wigfield, 1998)

Los experimentados caracterizan los valores como las actitudes de los/as estudiantes ante actividades de aprendizaje sobre dominios específicos (Psicología clínica, organizacional y laboral, forense y/o educacional) que devienen de una motivación intrínseca, de sus ideas en torno de los beneficios que implica su involucramiento en éstas. (Brophy, 1999)

Al mismo tiempo, sostienen que las creencias motivacionales operan como mediadoras potenciales de los aprendizajes. A modo de ejemplo, relatan las referidas a la auto-eficacia y autoestima que detectan ante las tareas asignadas. Especifican que la primera es la toma de conciencia por parte de los alumnos de sus capacidades para organizar y ejecutar acciones en pos de las metas (Zimmerman, 2000) y que, la segunda, a la eficacia personal suma la auto-confianza, el convencimiento de ser competentes para actuar.

Para los Psicólogos educativos, los estudiantes con auto-creencias positivas tienen metas académicas más desafiantes y muestran menor ansiedad a la vez que disfrutan de las actividades de aprendizaje que ejecutan. (Nieswandt, 2007)

Los y las docentes expresan que la motivación conjugan intereses personales, situacionales (situaciones de enseñanza) y por los tópicos de enseñanza. El último, estimulado por el Conocimiento Didáctico del Contenido (CDC) de los/as profesores/as, por su pasión por la enseñanza.

\section{Relaciones interpersonales con los estudiantes}

Consideran que las buenas prácticas de enseñanza requieren de profesores comprometidos y responsables con esta profesión. Hay que tener un amplio dominio de la disciplina, ser experto en lo que se enseña, tener un manejo pedagógico adecuado para interesar y despertar el gusto por la materia, fomentar y mantener buena relación con los estudiantes y tener objetivos claros.

Igualmente, ser autocríticos y formar alumnos críticos que pongan en cuestión saberes y conocimientos. 


\section{III.2. Pensamiento didáctico}

Los y las docentes experimentados manifiestan que con la experiencia, ciertos saberes sobre las prácticas docentes se asientan como fruto de sus ensayos y errores. Durante el proceso de aprendizaje, éstos/as elaboran recursos experienciales (Delory - Momberger, 2014) propios, algunos de los cuales constituyen un saber tácito. Éste, sirve de anclaje al reflexivo, cuya función es correctiva. (Sennet,2009)

Las sub-dimensiones del pensamiento del profesor refieren a las concepciones, creencias, visiones, imágenes acerca de la enseñanza, el aprendizaje y la evaluación

\section{Concepciones de enseñanza}

Identificamos un espectro de concepciones acerca de la enseñanza en la muestra de docentes participantes en el estudio, dando cuenta de la diversidad de significados y sentidos propuestos por Davini (2009).

En general, los docentes noveles y experimentados de cátedras del ciclo Orientado concuerdan con la idea de enseñanza como transmisión de conocimientos. Flexibilizan esta imagen, la noción de enseñanza como mecanismo para promover el crecimiento y el desarrollo personal de los estudiantes, con énfasis en la dimensión socio-afectiva:

Al enseñar es importante saber transmitir los contenidos de la materia y tener en cuenta que abonamos al desarrollo personal de quienes aprenden por lo que la relación afectiva, el clima agradable de la clase es fundamental (FB).

Profesores/as experimentados de materias del Ciclo de Profesionalización ( $3^{\circ}$ a $5^{\circ}$ años) y del área de la Formación Profesional comparten concepciones relacionadas a la enseñanza como: a) organización de las actividades que implican la participación activa de estudiantes a través de diversas estrategias didácticas y b) promoción de aprendizajes conducentes a modificar y/o enriquecer las ideas implícitas.

Dado que enseño a futuros Psicólogos lo hago proponiendo actividades dinámicas que los involucre y los ponga en situación de resolver casos o de aplicar la teoría en la interpretación de casos para que avancen desde sus ideas espontáneas a las científicas (RV).

A su vez, los profesionales docentes vinculados con las prácticas profesionales agregan la visión de la enseñanza como adiestramiento en habilidades, destrezas y adquisición de competencias profesionales.

Enseñarles a ser profesionales significa enseñar competencias propias del campo de la práctica profesional, tienen que saber, saber hacer y saber actuar (AM).

La mayoría de los/as profesores/as experimentados de asignaturas del ciclo de Profesionalización coincide con la idea de enseñanza como compartir y transmitir conocimientos y experiencias con las que sus estudiantes se van a encontrar en la futura vida profesional, trabajando con problemas y casos.

La enseñanza consiste en enfrentar a problemas y casos de la futura práctica profesional,...sólo de este modo van integrando conceptos, teorías, técnicas diversas...soy de la idea que se aprende a hacer, haciendo con la guía u orientación del profesor... (PNO). 
Los dos profesionales de mayor antigüedad, estiman que en tanto docentes actúan como mediadores entre el contenido y los estudiantes y pretenden proporcionar andamios para la apropiación de los conocimientos de sus disciplinas, desde un enfoque didáctico problematizador. Asimismo, asumen una perspectiva crítico-reflexiva en relación a la realidad del campo laboral y toman decisiones acerca de lo es epistemológicamente relevante en el trayecto formativo de los estudiantes de la carrera (Ortiz, 2002; Alcalá, 2002).

En este panorama de diversidad semántica, la enseñanza implica más que conocer y dominar técnicas, procedimientos y medios, requiere tomar conciencia de la complejidad de esta práctica sustantiva que caracteriza el "ser docente"- lo diferencia del experto en la disciplina o profesión- y confiere capacidad para la toma de decisiones fundadas.

\section{Concepciones de aprendizaje}

Los ingresantes tienen que aprender el oficio del estudiante en el ámbito universitario y no sólo introducirse en campos de conocimientos académicos:

Los alumnos aprenden dos cosas, a ser estudiantes universitarios y de modo activo a interpretar los objetos de estudio de las disciplinas psicológicas...me doy cuenta de que lo lograron cuando logran aplicar lo aprendido en una nueva situación. $(A R R)$

Dicho aprendizaje es un proceso de enculturación por el cual los estudiantes de la carrera se integran de manera gradual a una cultura de prácticas sociales (Díaz Barriga Arceo, 2003).

Por otro lado, en concordancia con concepciones de enseñanza como confrontación con el saber y aportar las ayudas para hacerlo propio (Meirieu, 2006) y/o capacidad de producir los efectos esperados de modo sostenido (Bunge y Ardila, 2002), los docentes expresan teorías constructivas del aprendizaje:

Cuando los alumnos aprenden hay un proceso de construcción de sus conocimientos desde lo que ya saben y sus experiencias previas (LG).

En este caso, se considera el papel del conocimiento pre-existente, es decir habilidades, creencias y conceptos que influyen significativamente en lo que perciben del medio, en el modo de organizar e interpretar el mundo.

Como profesora pretendo que aprendan a aprender, a comprender las teorías que necesitan para resolver los casos de la práctica de la profesión (RA).

Se alude al proceso de reestructuración, al conocimiento como interpretación de la experiencia que promueve y a la vez limita los procesos de construcción de significados (Putnam y Borko, 2000: 225). Los esquemas operan a manera de filtros de las experiencias y promover su re-elaboración es una condición necesaria para que los/as estudiantes comprendan, es decir, aprendan en profundidad (Entwistle, 1988).

Pienso que un buen profesor se caracteriza por su pericia al ponerlos en situación en una clínica, empresa, equipo interdisciplinario, y consigue que alumnos alcancen significativos logros académicos, adquieran un aprendizaje profundo y significativo y estén satisfechos con la enseñanza de sus docentes (OCM). 
Esta idea es consistente con lo sostenido por Hativa (2000) y Kane, et al., (2002) respecto de la forma-contenido para alcanzar resultados de calidad. Hablamos de un aprendizaje situado en el que los significados construidos dependen de la actividad que se realice, del contexto y de la cultura de pertenencia (Díaz Barriga Arceo, 2003).

En este sentido "La forma en que una persona aprende un conjunto concreto de conocimientos y habilidades, y... la situación en la que aprende... se convierten en partes fundamentales de lo que se aprende" (Putnam y Borko,2000: 257).

\section{Concepción de evaluación}

De acuerdo con los docentes, la evaluación incluye tres cuestiones: los aprendizajes de los estudiantes, los procesos de enseñanza y la programación de la materia.

Los fragmentos seleccionados refieren a la evaluación de los aprendizajes como parte del proceso de interacción entre profesores/as y alumnos, estructurada con la enseñanza y el aprendizaje a modo de mecanismo interno de control (Camilloni et al, 1998):

Al evaluar puedo analizar si avanzaron en el proceso y cuáles son sus logros, puedo controlar si alcanzaron los objetivos que propuse al planificar (PNO).

Busco que aprendan a evaluarse entre ellos, sus habilidades y conocimientos porque así trabajarán a futuro $(R A)$.

También aparece como instancia de apropiación de contenidos que informa sobre el proceso y ayuda a avanzar (Anijovich, 2017)

Es el momento en que puedo tener una idea de sus aprendizajes de los conocimientos y procedimientos propios de cada campo de la Psicología...me ayuda a ver si debo retomar algo que ofrece dificultades o puedo avanzar con mi programación $(A R)$.

Estas ideas dan cuenta de evaluación formativa, de instancias didácticas que proveen información para ser usada como feedback para modificar y mejorar las actividades de enseñanza y aprendizaje (Paul Black and Dylan Wiliam, 1998, citados por Camilloni). Cumple su función cuando los/as alumnos/as reciben información sobre su desempeño y orientaciones de cómo mejorarlo resolviendo sus dificultades y errores.

Es inherente a los procesos de enseñanza y aprendizaje y su finalidad es decidir acerca de la aprobación de los estudiantes asignándoles una calificación:

La evaluación es una pata de la enseñanza y del aprendizaje y mediante ella calificamos a los estudiantes para certificar si adquirieron los conocimientos de la Psicología para poder trabajar en el futuro (RV).

La concepción de evaluación, su sentido pedagógico -de acuerdo con Álvarez Méndez-, el del acto de conocimiento y ejercicio de la acción ética:

Pienso que la evaluación para calificar los aprendizajes es un encuentro con mis alumnos para ver qué aprendieron y ver a la vez qué aprendo yo con cada grupo...me sirve para saber cuáles son sus conocimientos y lo que no saben todavía... (ARR). 
Lo difícil es ser objetivos y justos...no es fácil evaluar lo que aprendieron, tal vez si darse cuenta de si leyeron y reproducen lo leído... (AM)

En este sentido, es más que una cuestión técnica centrada en la objetividad, es además, un asunto de ética focalizada en la acción justa.

Además de esta perspectiva de la evaluación centrada en la dimensión ética, es decir servicio de los aprendizajes (por qué y para qué evaluar), emergen concepciones con foco en la selección social, esto es, en el control y la medición:

Tenemos que evaluar para certificar quiénes realmente saben de esta profesión, tenemos que calificarlos y aquellos que no alcanzan los conocimientos y habilidades que se requieren, deben recursar la materia $y$ a veces pensar si es esto lo que realmente quieren (MA).

Asimismo, para los estudiantes lo que forma parte del examen o instancia de evaluación es válido, lo que debe aprender y cómo lo debe hacer, por eso, la evaluación legitima saberes y configura aprendizajes, tal como se evidencia en el siguiente testimonio:

Siempre antes de evaluar digo a mis alumnos qué deben saber, qué tienen que estudiar cómo tienen que estudiar para resolver las situaciones que voy a plantear, destaco lo más importante que deben saber (LG).

Los diversos constructos dan cuenta de la evaluación de los procesos de aprendizaje para conocer los logros de cada estudiante, calificarlo y promoverlo; ayudarlo en el proceso de aprendizaje y además, de la calidad de la docencia.

La evaluación cumple una función pedagógica, se vincula con los procesos de formación de los sujetos y no se agota en la acreditación ni en la calificación de un examen.

La primera constituye la necesidad institucional de certificar el aprendizaje de conocimientos curricularmente previstos y de asignar una calificación conforme escalas predeterminadas para la promoción o no del estudiante de acuerdo con el Régimen pedagógico de la unidad académica.

\section{Lo que dicen que hacen los profesores}

Los profesores manifiestan acordar con sus pares académicos de otras sedes los núcleos básicos y derivados de los contenidos, el tipo de actividades de aprendizaje y los modos de evaluación y cronograma de trabajos prácticos y de evaluaciones, antes de planificar sus proyectos pedagógicos. Estos acuerdos se registran en un acta que se entrega a la Coordinación.

De acuerdo con los lineamientos institucionales:

- Elaboran la planificación sobre la base de una grilla institucional que consta de dos partes: 1) diseño del proyecto y 2) evaluación de la propuesta didáctica. La primera se entrega al inicio del dictado y ambas, al finalizar el cursado.

- Evalúan mediante dos trabajos prácticos, un examen parcial escrito individual, un coloquio integrador final y una nota conceptual en torno a diferentes competencias que deben desarrollar y acreditar los estudiantes al finalizar el cursado de cada materia. Las competencias se discriminan en transversales y específicas con diferente nivel o grado de complejidad por ciclo.

- Entregan algunos ejemplares de los trabajos prácticos, la totalidad de trabajos del examen parcial y ejemplos del coloquio integrador. 
Ejemplificamos los acuerdos entre docentes de dos sedes en una de las cátedras de $2^{\circ}$ año de la Licenciatura en Psicología.

En la fundamentación exponen en primer lugar, los aportes de la materia a la formación del Licenciado en Psicología, fundamentos teóricos que le permitirán una toma de posición respecto a su futura práctica profesional y son a la vez, un saber propedéutico para el abordaje de cátedras posteriores.

Además, señalan las articulaciones verticales con materias de $1^{\circ}, 3^{\circ}, 4^{\circ}$ y $5^{\circ}$ años, re-significando y ampliando los conocimientos previos adquiridos en $1^{\circ} \mathrm{y}$ abordando las teorías en sus contextos de origen y evolución.

Los objetivos apuntan a la comprensión de fundamentos y principios, a la identificación de conceptos tensores en la evolución de teorías, al desarrollo del juicio crítico respecto de las teorías, a afianzar el vocabulario técnico y a la articulación de los contenidos de la materia con otras áreas del conocimiento y la cultura.

Presentan los contenidos organizados en unidades que contienen los núcleos básicos y derivados, y consignan los objetivos específicos y la bibliografía por cada unidad.

Entre las estrategias de enseñanza proponen: análisis de casos, talleres de discusión y de lectura, exposición dialogada y clases especiales.

Los talleres de discusión se realizan con documentales, extractos de películas; casos clínicos con la finalidad de relacionar la teoría y la práctica, estimular el pensamiento reflexivo, la discusión crítica y el desarrollo de hábitos de expresión oral.

En los talleres de lectura se trabajan los textos más complejos y que generan dificultades de comprensión en base a guías e intercambios grupales.

También, analizan casos paradigmáticos de cada uno de los exponentes de las teorías y en las clases especiales, profundizan algunos temas que exponen oralmente en grupos.

De acuerdo con el régimen pedagógico pactan el cronograma de evaluación del único examen parcial, de los dos trabajos prácticos y del trabajo integrador final, de los cuales entregan evidencias a las autoridades.

Los exámenes parciales son útiles para las autoridades, brindan información general y permiten diagnosticar errores más frecuentes. La suma de éstos, de los trabajos prácticos e integrador final, más la nota conceptual de las competencias desarrolladas constituyen la evaluación certificadora que permite observar y evaluar los logros finales de un proceso de aprendizaje, otorgar una calificación y decidir la promoción. A la vez, facilitan la comparación alumnos y docentes

Señalan que seleccionaron los instrumentos en función de las estrategias didácticas y actividades de aprendizaje acordadas, de los contenidos y de los objetivos propuestos.

Entre las actividades de modalidad oral, figuran exposición de temas, debates, presentaciones y defensas de trabajos; de modalidad escrita, pruebas semiestructuradas; resolución de situaciones problemáticas, portafolios e informes de casos.

También acuerdan la modalidad de evaluación del proceso basada en la observación sistemática mediante fichas de seguimiento y lista de cotejo o control.

Además, intercambian ideas acerca de otras actividades de gestión curricular en relación con sus espacios:

a) visitas a instituciones vinculadas directamente con los contenidos de las cátedras o con actividades del campo de la práctica profesional;

b) conferencia de especialistas invitados;

c) actividades inter-cátedras

Expresan también acordar la planificación y ejecución de actividades de investigación y de extensión. Ambos tipos de actividades son remuneradas por la unidad académica. 
Respecto de las primeras, pactan temas u objetos-problemas para trabajar en cada sede o nodo articulado inter-sedes.

Generalmente, las actividades de extensión se concretan en cada sede conforme la disponibilidad temporal de los docentes.

\section{Conclusiones}

Generalmente, la falta de una formación pedagógica sistemática de los/as Licenciados/as en el ámbito universitario conduce a pensar que sus intervenciones áulicas adolecen de esta dimensión del CPD. Sin embargo, éstos/as no enseñan las materias de la carrera como las estudiaron o como la conocen los/as especialistas en la disciplina. De modo consciente o inconsciente adaptan, reconstruyen, transforman y simplifican el contenido para hacerlo comprensible a sus estudiantes. Esto es, construyen y re-construyen Conocimiento Didáctico del Contenido (CDC) en la praxis docente.

Las y los profesores participantes en el estudio destacados por los/as estudiantes-dan cuenta del enriquecimiento de este constructo en términos de hipótesis de progresión. Particularmente, algunos experimentados/as, develan experticia en función del impacto de sus intervenciones en los procesos de enseñanza y aprendizaje y espacios de formación en el oficio del Psicólogo.

EI CDC de los casos analizados revela un continuum de concepciones y posicionamientos en torno a los componentes de la triada didáctica, lo que permite inferir que la estrategia institucional de acuerdos y planificación entre pares de las diferentes sedes coadyuva -en parte- a la mejora de las prácticas profesionales docentes.

El estudio aporta una aproximación al constructo y se constituye en un insumo significativo para repensar las líneas de acción de la formación docente continua.

\section{Referencias Bibliográficas}

Alcalá, María Teresa. (2002). El conocimiento del profesor y enfoques didácticos. Ficha de cátedra. Facultad de Humanidades. UNNE. pp. 1-29.

Anijovich, R. (2013). Profesores reflexivos para "repensar lo pensado". Suplemento educativo del Diario Clarín, Buenos Aires, 25 de marzo de 2013. Recuperado de: http://www.clarin.com/educacion/Profesores-reflexivos-repensarpensado $0890311172 . \mathrm{html}$

Anijovich, R y Cappelletti,G. ( 2017) La evaluación como oportunidad. Buenos Aires: Paidós.

Álvarez, Z. \& Sarasa, M. C. (2007). Una mirada a la buena enseñanza desde los relatos de profesores y alumnos. En M. Borgström \& L. Porta (Eds.). La formación de profesores en Suecia y Argentina: lecturas y perspectivas convergentes (pp. 107132). Edición bilingüe español-inglés. Mar del Plata: GIEEC, Facultad de Humanidades, Universidad Nacional de Mar del Plata

Bain, K. (2007). Lo que hacen los mejores profesores universitarios; (2da. Ed.). Valencia: PUV. Cap. 3 y 5.

Press

Bain, K. (2004), What the best College Teachers do?, USA, Harvard University

Bain, Ken. (2012). “¿Qué es la buena enseñanza?”. Revista de Educación, Número 4, Año 3.Facultad de Humanidades. UNMDP. pp. 63- 74

Benavente, A. (2007). Good Practice: an example to prove the rule or a lighthouse to guide our steps. Informe presentado al 5o Seminario Internacional del BIE/UNESCO sobre "Innovations curriculaires et lutte contre la pauvertè: les rôles cruciaux des écoles, des enseignants et des communautés pur la mise en oeuvre du curriculum". Junio 2007. Extraído el 13 de marzo de 2012.

http://www.ibe.unesco.org/fileadmin/user_upload/Poverty_alleviation/Seminair 
es/5eSeminaire_Maurice_juin07/5eSeminaire_Maurice_juin07_Annexe6_EN.pd

Brophy, J. (1999). Hacia un modelo de los aspectos de valor de la motivación en la educación: Desarrollo del aprecio por ... Psicólogo educativo, 34 (2), 75-85. Editores

Bunge, M. y Ardila R. (2002), Filosofía de la psicología, México: Siglo XXI

Camilloni A.E.W. de, Celman S.C., Litwin E.y Maté M.C. P. de (1998) La evaluación de los aprendizajes en el debate didáctico contemporáneo. Buenos Aires: Paidós.

Carlos Guzmán (2009) ¿Cómo enseñan psicología los profesores efectivos? Un estudio exploratorio, Perfiles Educativos / Tercera época, vol. XXX, núm. 123, 2009 / IISUE-UNAM

Davini, M. C. (2009). Métodos de enseñanza. Didáctica general para maestros $y$ profesores; (1ra. reimp.). Buenos Aires: Santillana.

Delory-Momberger, C. (2014). Experiencia y formación. Biografización, biograficidad y heterobiografía. Revista Mexicana de Investigación Educativa, 19(62), 695-710.

Díaz Barriga Arceo, F. (2003). "Cognición situada y estrategias para el aprendizaje

significativo". En Revista electrónica de investigación educativa, vol. 5, № 2 . http://redie.uabc.mx. 10 pág

Diker, G. y Terigi, F. (1997). La formación de maestros y profesores. Hoja de ruta. Buenos Aires: Paidós.

Eccles, JS, Wigfield, A, Schiefele U. 1998b. Motivation. See Eisenberg 1998, pp. 1017-95 Paidós.

Entwistle, N. (1988). La comprensión del aprendizaje en el aula. Buenos Aires;

Feldman, D. (1999) Ayudar a enseñar. Aiqué. Buenos Aire

sobre la

Fenstermarcher, G. (1989). "Tres aspectos de la filosofía de la investigación

enseñanza". En Wittrock, Merlin. La investigación de la enseñanza, I. Buenos Aires:

Paidós. profesión.

Fernández Pérez, J. (2001). Elementos que consolidan el concepto de Notas para su reflexión. Revista Electrónica de Investigación Educativa, 3 (2). En: http://redie. Uabc.mx/vol3 no2/contenidofernandez.html.

Freud, S. (1892-1893). Sobre la psicología del colegial. Obras completas Tomo 2. Madrid: Biblioteca Nueva.

Hativa, N. (2000), Teaching for Effective Learning in Higher Education, Dordrecht/ Boston/Londres, Kluwer Academic Publishers

Jackson, P. (2002). Práctica de la enseñanza. Buenos Aires: Amorrortu

Review

Kane, R., S. Sandretto y Ch. Heath (2002), "Telling Half of the Story: A Critical

of Research on the Teaching Beliefs and Practices of University Academics", Review of Educational Research, verano, 72 (2), 177-228

Korthagen, F. (2001), Linking Practice and Theory. The Pedagogy of Realistic Teacher Education, USA, Lawrence Erlbaum Associates

Litwin, E. (2008). El oficio de enseñar. Condiciones y contextos. Buenos Aires: Paidós.

Marcelo García, C. (2009). Los comienzos en la docencia: un profesorado con buenos principios. Profesorado. Revista de Curriculum y Formación del Profesorado, $13(1), 1-25$. 
Medina Moya, J. (2006). La profesión docente y la construcción del conocimiento profesional. Buenos Aires: Lumen.

Graó

Meirieu, P. (2006), Carta a un joven profesor. Por qué enseñar hoy, Barcelona,

Nieswandt, M. (2007). Student Affect and Conceptual Understanding in Learning Chemistry. Journal of Research in Science Teaching, 44(7), 908-937.

Ortiz, M.C Concepciones epistemológicas y pedagógico-didácticas de los formadores de formadores en torno a la formación docente. Tesis Maestría. Facultad Humanidades, UNNE, 2002.

Freire,P. (1970).Pedagogìa del oprimido.México: Siglo XXI.2da.ed.

Porta, L.y Sarasa, M.C. (Comp.). (2008). "Introducción", en Formación y desarrollo de la profesión docente en el profesorado: las buenas prácticas y sus narrativas". Facultad de Humanidades. UNMDP. pp. 7-22.

Putnam, R. T. y Borko, H. (2000). "El aprendizaje del profesor: implicaciones de las nuevas perspectivas de la cognición". En Biddle, Bruce J.; Good, Thomas L. y Goodson, Ivor F. La enseñanza y los profesores I. La profesión de enseñar. Barcelona: Paidós. pp. 219-309.

Sánchez Blanco, Gaspar Reseña de "Conceptos claves en la didáctica de las disciplinas" de Jean P. Astolfi Revista Interuniversitaria de Formación del Profesorado, vol. 17, núm. 1, 2003, pp. 225-226 Zaragoza, España, Universidad de Zaragoza

Disponible en: http://www.redalyc.org/articulo.oa?id=27417125

Stake, R. (1994). Case Studies. En N. Denzin; Y. Lincoln (Eds.), Handbook of Qualitative Research (pp. 236-247). California: Sage.

Sennett, R. (2009). El artesano. Barcelona: Anagrama.

Shulman, L. (1987). Knowledge and teaching. Harvard Educational Review, $57(1), 1-21$

Shulman, L. (1986). Those who understand: knowledge growth in teaching. Educational Researcher, 15(2), 4-14.

Schön, D. (1982). El profesional reflexivo. Cómo piensan los profesionales cuando actúan. Buenos Aires: Paidós.

Schön, D. (1992). La formación de profesionales reflexivos. Barcelona: Paidós

Sanjurjo, L. (2004). La construcción del conocimiento profesional docente en La Formación docente. Publicación de Conferencias y Paneles del 2do. Congreso Internacional de Educación. (pp. 121-129). Santa Fe: Universidad Nacional del Litoral

Sanjurjo, L. (2012). "La clase: un espacio estructurante de la enseñanza". Revista de Educación, Año 2, Nro. 3. Facultad de Humanidades de la Universidad Nacional de Mar del Plata. EUDEM. pp. 71-84

Torres,R.M (1998) ¿Qué y cómo es necesario aprender? Necesidades básicas de aprendizaje y contenidos curriculares. México: SEP.

Van Haecht, A. (1998): La escuela va a examen. Madrid, España: Biblos.

Zabalza, M. (2006). La convergencia como oportunidad para mejorar la docencia universitaria. Revista Interuniversitaria de Formación del profesorado, 20 (3), 17-35.

Zimmerman, M. (2000). Empowerment theory. En J. Rappaport \& E. Seidman (Eds). Handbook of community psychology (pp. 43-63). New York, NY: Kluwer. 